\title{
A New Fuzzy DEA Model for Solving the MCDM Problems in Supplier Selection
}

Chi-Mao Tsai

Department of Shipping and Transportation Management, National Taiwan Ocean University, Keelung 20224, Taiwan, $R O C$

Hsuan-Shih Lee

Department of Shipping and Transportation Management, National Taiwan Ocean University, Keelung 20224, Taiwan, ROC, hslee@email.ntou.edu.tw

Guo-Ya Gan

College of Auditing and Evaluation, Nanjing Audit University, Nanjing, Jiangsu 211815, China, ganguoya@foxmail.com

Follow this and additional works at: https://jmstt.ntou.edu.tw/journal

Part of the Fresh Water Studies Commons, Marine Biology Commons, Ocean Engineering Commons, Oceanography Commons, and the Other Oceanography and Atmospheric Sciences and Meteorology Commons

\section{Recommended Citation}

Tsai, Chi-Mao; Lee, Hsuan-Shih; and Gan, Guo-Ya (2021) "A New Fuzzy DEA Model for Solving the MCDM Problems in Supplier Selection," Journal of Marine Science and Technology. Vol. 29: Iss. 1, Article 7.

DOI: 10.51400/2709-6998.1006

Available at: https://jmstt.ntou.edu.tw/journal/vol29/iss1/7

This Research Article is brought to you for free and open access by Journal of Marine Science and Technology. It has been accepted for inclusion in Journal of Marine Science and Technology by an authorized editor of Journal of Marine Science and Technology. 


\title{
A New Fuzzy DEA Model for Solving the MCDM Problems in Supplier Selection
}

\author{
Chi-Mao Tsai ${ }^{a}$, Hsuan-Shih Lee ${ }^{a, b, *}$, Guo-Ya Gan ${ }^{\mathrm{c}, * *}$ \\ ${ }^{a}$ Department of Shipping and Transportation Management, National Taiwan Ocean University, Keelung 20224, Taiwan, ROC \\ ${ }^{\mathrm{b}}$ Department of Information Management, Ming Chuan University, Taipei 11103, Taiwan, ROC \\ ${ }^{c}$ College of Auditing and Evaluation, Nanjing Audit University, Nanjing, Jiangsu 211815, China
}

\begin{abstract}
Supplier selection is a crucial task in supply chain management, and good supply chain management is essential to a company's ability to develop sustainably and gain the trust and satisfaction of its customers. In modern supply chain management, supplier selection often takes the form of a multi-criteria decision-making (MCDM) problem. Thus, to solve this problem, this study formulated a novel fuzzy MCDM model, which is based on the fuzzy data envelopment analysis model. This novel approach can transform the linguistic variables of assessment criteria into well-founded quantitative data for calculation. Moreover, our approach can effectively discriminate between decision-making units (both efficient and inefficient) to aid managers in identifying the most suitable suppliers. We hope that our model can provide scholars and managers with new insights into solving the MCDM problem in supplier selection.
\end{abstract}

Keywords: Data envelopment analysis (DEA), Fuzzy MCDM, Supplier selection, Sustainable development

\section{Introduction}

I n modern supply chain management, ideas and methods in integrated management have been employed to efficiently connect together each enterprise in the production process; this has been executed in conjunction with advances in digitization, visualization, and intelligent science and technology. Such connectivity enables the coordinated development of procurement, production, and sales; it helps the entire supply chain be more efficient and the whole system be more cost effective. At present, the field of modern supply chain management is focusing on such an integration of suppliers, manufacturers, warehouses, distribution centers, and distributors into an organic whole. In the context of these developments, effective operation management determines how enterprises formulate their overall strategic deployment and operations, playing a crucial role in making enterprises more competitive in the international market. Because of rapid developments in science and technology, the primary concern of modern supply chain management is to select the most suitable raw material suppliers through scientific methods and not simply to minimize transportation costs during the production process or reduce the corresponding inventory of each link. In selecting the most suitable suppliers, the entire supply chain system is rendered more cost efficient, and the company can also satisfy actual customer demands by producing and operating more efficiently. However, many enterprises have faced impediments to efficient operation and cost management, particularly from the complexity of raw material procurement in relation to production tasks. In particular, enterprise managers

Received 22 June 2020; revised 7 July 2020; accepted 4 August 2020.

Available online 31 March 2021

* Corresponding author.

** Corresponding author.

E-mail addresses: hslee@email.ntou.edu.tw (H.-S. Lee), ganguoya@foxmail.com (G.-Y. Gan).

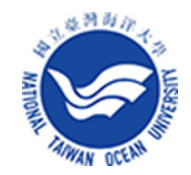


must consider various raw material supplier-related criteria (such as price, product quality, and after-sales service quality) in selecting the best suppliers that deliver the best quality and most efficient performance for their price.

Supplier selection is a typical multi-criteria decision-making (MCDM) problem. In particular, an evaluation is an MCDM problem if it involves (a) two or more decision objectives and (b) multiple criteria. Decision-making analysis pertains to the process of deciding on the best solution, among a set of alternatives, to solve current or future problems; in doing so, planning, design, and manufacturing is considered jointly in the evaluation system.

For enterprises, strategic decision-making is complex because various criteria must be considered. For example, when deciding on whether to embark on a major technological transformation project, the company must consider, for example, production safety and environmental protection as well as the corresponding benefits to society and to the company's profit margin. In addition, various criteria must be selected to optimize the evaluation program in pursuit of these objectives. To handle such complexity, MCDM methods have been widely applied in various fields-such as supply chain management, water resource utilization, process design, green energy, and economic policymaking. Some commonly used MCDM methods include the analytic hierarchy process (AHP) [1,2], multi-objective fuzzy method [3-5], multi-objective grey wolf optimizer [6,7], the technique for order of preference by similarity to ideal solution (TOPSIS) method [8,9], stochastic frontier analysis (SFA) $[10,11]$, the hull free disposal method (FDH) [12,13], and data envelopment analysis (DEA) [14-17].

Supplier selection, being an important MCDM problem, has been studied for decades. Lee [18] proposed an innovative model based on fuzzy theory to deal with the interval-valued fuzzy number; this model allows for the supplier selection problem to be solved in cases where uncertainty exists.Fu [19] integrated the AHP method with additive ratio assessment to deal with the MCDM problem of selecting catering suppliers in the airline industry. Furthermore, considering the increasing importance of environmental protection, Haeri and Rezaei [20] proposed a novel greybased model that accounts for both economic and environmental criteria in green supplier selection. Similarly, Kilic and Yalcin (2020) [21] also emphasized the importance of environmental factors in supplier selection. They combined intuitionistic fuzzy TOPSIS and a modified fuzzy goal programming model to manage the selection problem in a multi-period environment. As a well-known nonparametric approach for evaluating the relative efficiency of each decisionmaking unit (DMU), DEA has also made much contribution to solving the supplier selection problem under a synthetic consideration of multiple inputs and outputs. For example,Dobos and Vörösmarty [22] distinguished factors into traditional (managerial) and environmental (green) factors and expanded the traditional criteria and weight system in supplier evaluation research. They did so by using a DEA approach with common weights analysis to identify a weight system in which environmental factors can influence the final supplier selection.

Furthermore, Jauhar and Pant [23] integrated the DEA with the DE algorithm to develop a new evaluation system for solving the problem of sustainable supplier selection. In their study, an example involving the actual automobile industry was analyzed to confirm the effectiveness of their new model. Dobos and Vörösmarty [24] further proposed a DEA-type supplier selection method for selecting the best green supplier. By considering the green factors as the outputs and the management variables as the inputs, they also examined the effect of inventory-related costs or ordering costs on the selected supplier. Davoudabadi et al. [25] noted that resilience has become an increasingly important criterion in this field of high quality supplier selection due to disruptions to the balance of cost, time, and quality in the interactions between developed and developing countries.

In general, supplier selection is an important MCDM problem for enterprises. The problem involves not only quantitative criteria but also various ambiguous factors, where some standards of many suppliers cannot be quantified with concrete data. Karsak and Dursun [26] addressed this problem by integrating DEA with quality function deployment in developing a novel fuzzy MCDM framework; their framework included factors that accounted for vagueness and imprecision. They verified their framework in a case study of a private hospital in Istanbul.Azadi et al [27] proposed a fuzzy theory-based model that integrated the DEA with the enhanced Russell measure. Their model was used to help select the best sustainable supplier for a resin production enterprise. They noted that their new model could evaluate the efficiency and productivity in an uncertain environment with different $\alpha$ levels-helping managers consider the social, economic, and environmental factors when selecting the best sustainable supplier. Furthermore, in the context of fuzzy multi-objective DEA, Cheng et al. [28] developed a hybrid DEA-AdaBoost model to meet the challenge of the emergence of big data. Their innovative model was constructed based on an expert's database, which contained a list of appropriate and inappropriate suppliers. Their simulation results 
demonstrated that their proposed model yielded improved accuracy and a reduced time of consumption for supplier selection. Rashidi and Cullinane, [29] utilized both fuzzy DEA and fuzzy TOPSIS models to produce a useful pooled shortlist of potential sustainable suppliers, where the database was derived from a logistics service provider in Sweden. Their results had provided a reference for companies when analyzing service quality commitments and negotiating prices with potential sustainable suppliers. In general, existing studies have integrated fuzzy theory and the DEA method to effectively convert linguistic variables into quantitative data for analytical evaluation in the process of supplier selection.

However, these proposed fuzzy DEA models have all been based on the traditional DEA model [30,31], which may lead to the identification of more than one optimal supplier. Thus, to resolve the MCDM problem in supplier selection, this study proposes a new fuzzy DEA model based on the super-efficiency DEA model.

The remaining parts of the paper are organized as follows. Section 2 presents the concept of a fuzzy number and the DEA. Section 3 presents the new proposed fuzzy MCDM model in multiplier form, which is based on super-efficiency DEA, in addition to the dual of the new model in envelopment form. Section 4 presents two numerical examples, which demonstrate the effectiveness of our model. Section 5 concludes the paper.

\section{Preliminaries}

Definition 1. A fuzzy set $\tilde{A}=\left\{\left(x, \mu_{\tilde{A}}(x)\right) \mid x \in U\right.$, $\left.0 \leq \mu_{\tilde{A}}(x) \leq 1\right\}$. Specifically, a fuzzy set is a set of pairs: one member of the pair is an element in the universe, and the other member of the pair is the element's degree of membership in the fuzzy set. Formally, membership in a fuzzy set $\tilde{A}$ is defined by the membership function $\mu_{\tilde{A}}: U \rightarrow[0,1]$. If $\mu_{\tilde{A}}: U \rightarrow$ $\{0,1\}$, then $\tilde{A}$ is a crisp set.

Definition 2. A fuzzy set $\tilde{A}$ is normal if $\sup _{x \in U} \mu_{\tilde{A}}(x)=$ A fuzzy set can be represented by a set of $\alpha$-cuts of the fuzzy set. The $\alpha$-cuts of a fuzzy set are defined as follows:

Definition 3. Consider the fuzzy set $\tilde{A}=\{(x$, $\left.\left.\mu_{\tilde{A}}(x)\right) \mid x \in U, 0 \leq \mu_{\tilde{A}}(x) \leq 1\right\}$. The $\alpha$-cuts of $\tilde{A}$ are defined to be $\tilde{A}^{\alpha}=\left\{x \mid \mu_{\tilde{A}}(x) \geq \alpha, x \in U\right\}$. The strong $\alpha$-cuts of $\tilde{A}$ are defined to be $\tilde{A}^{\alpha+}=\left\{x \mid \mu_{\tilde{A}}(x)>\alpha\right.$, $x \in U\}$.

A fuzzy number of is a convex fuzzy set on real number $R$. The convex fuzzy set is defined as follows:
Definition 4. $\tilde{A}=\left\{\left(x, \mu_{\tilde{A}}(x)\right) \mid x \in R, 0 \leq \mu_{\tilde{A}}(x) \leq 1\right\}$ is said to be convex if it is the case that if $x_{1} \leq x_{2}$, then $\mu_{\tilde{A}}\left(t x_{1}+(1-t) x_{2}\right) \geq \min \left(\mu_{\tilde{A}}\left(x_{1}\right), \mu_{\tilde{A}}\left(x_{2}\right)\right)$, where $0 \leq t \leq 1$. A triangular fuzzy number is defined as follows:

Definition 5. A fuzzy set $\tilde{A}$ is a triangular fuzzy number if it is a normal fuzzy number that satisfies the following equality:

$\mu_{\tilde{A}}(x)=\left\{\begin{array}{l}\frac{x-a_{1}}{a_{2}-a_{1}}, a_{1} \leq x \leq a_{2} \\ \frac{a_{3}-x}{a_{3}-a_{2}}, a_{2} \leq x \leq a_{3} . \\ 0, \text { otherwise }\end{array}\right.$.

A triangular fuzzy number can be represented as $\tilde{A}=\left(a_{1}, a_{2}, a_{3}\right)$.

An MCDM problem is one where there are $n$ alternatives under consideration against $m$ criteria. This problem can be represented by a decision matrix $D$, which is given as:

$D=\left[\begin{array}{cccc}A_{11} & A_{12} & \cdots & A_{1 m} \\ A_{21} & A_{22} & \cdots & A_{2 m} \\ \vdots & \vdots & \cdots & \vdots \\ A_{n 1} & A_{n 2} & \cdots & A_{n m}\end{array}\right]$

where $A_{i j}$ represents the rating of alternative $i$ against criterionj. A MCDM problem is defined as the aggregation of the information in $D$ such that alternatives can be ranked appropriately. If the rating $A_{i j}$ is a fuzzy number, the problem is a fuzzy MCDM problem, which is the type of problem considered in this paper. Instead of aggregating the fuzzy decision matrix, which is the usual approach [29,32-36], we proposed a method based on DEA. DEA is a nonparametric method pioneered by Charnes et al., [31] for evaluating DMU performance. The DEA proceeds as follows. Consider $n$ DMUs to be evaluated, where each DMU is associated with $m$ inputs and $s$ outputs. The $i$ th input of DMU $j$ is denoted as $x_{i j}$, and the $r$ th output of DMU $j$ is denoted as $y_{r j}$. The constant returns to scale (CRS)-based multiplier form, first proposed by Charnes et al., [31]; is as follows:

$\max \sum_{r=1}^{s} u_{r} y_{r k}$

s.t.

$\sum_{i=1}^{m} v_{i} x_{i k}=1$

$\sum_{r=1}^{s} u_{r} y_{r j}-\sum_{i=1}^{m} v_{i} x_{i j} \leq 0, j=1, \ldots, n$

$v_{i} \geq 0, i=1, \ldots, m$

$u_{r} \geq 0, r=1, \ldots, s$ 
where $v_{i}$ and $u_{r}$ are the weights of the $i$ th input and $r$ th output respectively.

\section{Fuzzy DEA model}

Consider $m$ cost criteria and sbenefit criteria. There criteria entail two decision matrices for the set of alternatives: the cost decision matrix and the benefit matrix, which are represented as follows.

$X=\left[\begin{array}{cccc}\tilde{x}_{11} & \tilde{x}_{12} & \cdots & \tilde{x}_{1 m} \\ \tilde{x}_{21} & \tilde{x}_{22} & \cdots & \tilde{x}_{2 m} \\ \vdots & \vdots & \cdots & \vdots \\ \tilde{x}_{n 1} & \tilde{x}_{n 2} & \cdots & \tilde{x}_{n m}\end{array}\right]$ and $Y=\left[\begin{array}{cccc}\tilde{y}_{11} & \tilde{y}_{12} & \cdots & \tilde{y}_{1 s} \\ \tilde{y}_{21} & \tilde{y}_{22} & \cdots & \tilde{y}_{2 s} \\ \vdots & \vdots & \cdots & \vdots \\ \tilde{y}_{n 1} & \tilde{y}_{n 2} & \cdots & \tilde{y}_{n s}\end{array}\right]$

Let $\tilde{x}_{i j}$ denote the fuzzy number for the rating for the $j$ th supplier on the $i$ th cost criterion where $1 \leq i \leq$ $m$. Let $\tilde{y}_{r j}$ denote the fuzzy number for the rating for the $j$ th supplier on the $r$ th benefit criterion, where $1 \leq r \leq s$. Assume that the fuzzy numbers for the ratings are triangular fuzzy numbers. Let $\tilde{x}_{i j}=\left(x_{i j}^{L}\right.$, $\left.x_{i j}^{M}, x_{i j}^{R}\right)$, where $x_{i j}^{L} \leq x_{i j}^{M} \leq x_{i j}^{R}$. Let $\tilde{y}_{r j}=\left(y_{r j}^{L}, y_{r j}^{M}, y_{r j}^{R}\right)$, where $y_{r j}^{L} \leq y_{r j}^{M} \leq y_{r j}^{R}$. Because the rating is a triangular fuzzy number, let the weight for $i$ th cost criterion be $v_{i}=\left(v_{i}^{L}, v_{i}^{M}, v_{i}^{R}\right)$ where $0 \leq v_{i}^{L}, v_{i}^{R} \leq v_{i}^{M}$. Likewise, let the weight for $r$ th benefit criterion be $u_{r}=\left(u_{r}^{L}, u_{r}^{M}, u_{r}^{R}\right)$, where $0 \leq u_{r}^{L}, u_{r}^{R} \leq u_{r}^{M}$. The aggregated score for the $k$ th supplier is:

$\frac{\sum_{r=1}^{s} u_{r} \cdot \tilde{y}_{r k}}{\sum_{i=1}^{m} v_{i} \cdot \tilde{x}_{i k}}=\frac{\sum_{r=1}^{s}\left(u_{r}^{L} y_{r k}^{L}+u_{r}^{M} y_{r k}^{M}+u_{r}^{R} y_{r k}^{R}\right)}{\sum_{i=1}^{m}\left(v_{i}^{L} x_{i k}^{L}+v_{i}^{M} x_{i k}^{M}+v_{i}^{R} x_{i k}^{R}\right)}$

The model for obtaining the best score for the $k$ th supplier is:

$\max \frac{\sum_{r=1}^{s}\left(u_{r}^{L} y_{r k}^{L}+u_{r}^{M} y_{r k}^{M}+u_{r}^{R} y_{r k}^{R}\right)}{\sum_{i=1}^{m}\left(v_{i}^{L} x_{i k}^{L}+v_{i}^{M} x_{i k}^{M}+v_{i}^{R} x_{i k}^{R}\right)}$

s.t.

$\frac{\sum_{r=1}^{s}\left(u_{r}^{L} y_{r j}^{L}+u_{r}^{M} y_{r j}^{M}+u_{r}^{R} y_{r j}^{R}\right)}{\sum_{i=1}^{m}\left(v_{i}^{L} x_{i j}^{L}+v_{i}^{M} x_{i j}^{M}+v_{i}^{R} x_{i j}^{R}\right)} \leq 0, j=1, \ldots, n$
Model (6) is in fractional programming, which can be further linearized into the following linear programming:

$\max \sum_{r=1}^{s}\left(u_{r}^{L} y_{r k}^{L}+u_{r}^{M} y_{r k}^{M}+u_{r}^{R} y_{r k}^{R}\right)$

s.t.

$\sum_{i=1}^{m}\left(v_{i}^{L} x_{i k}^{L}+v_{i}^{M} x_{i k}^{M}+v_{i}^{R} x_{i k}^{R}\right)=1$

$$
\begin{aligned}
& \sum_{r=1}^{s}\left(u_{r}^{L} y_{r j}^{L}+u_{r}^{M} y_{r j}^{M}+u_{r}^{R} y_{r j}^{R}\right) \\
- & \sum_{i=1}^{m}\left(v_{i}^{L} x_{i j}^{L}+v_{i}^{M} x_{i j}^{M}+v_{i}^{R} x_{i j}^{R}\right) \leq 0, j=1, \ldots, n
\end{aligned}
$$

$0 \leq v_{i}^{L}, v_{i}^{R} \leq v_{i}^{M}, 1 \leq i \leq m$

$0 \leq u_{r}^{L}, u_{r}^{R} \leq u_{r}^{M}, 1 \leq r \leq s$

The score obtained by model (7) is less than or equal to 1 . To enhance the discrimination power, we remove such a restriction. The resulting model is as follows:

$\max \sum_{r=1}^{s}\left(u_{r}^{L} y_{r k}^{L}+u_{r}^{M} y_{r k}^{M}+u_{r}^{R} y_{r k}^{R}\right)$

s.t.

$\sum_{i=1}^{m}\left(v_{i}^{L} x_{i k}^{L}+v_{i}^{M} x_{i k}^{M}+v_{i}^{R} x_{i k}^{R}\right)=1$

$$
\begin{aligned}
& \sum_{r=1}^{s}\left(u_{r}^{L} y_{r j}^{L}+u_{r}^{M} y_{r j}^{M}+u_{r}^{R} y_{r j}^{R}\right) \\
& -\sum_{i=1}^{m}\left(v_{i}^{L} x_{i j}^{L}+v_{i}^{M} x_{i j}^{M}+v_{i}^{R} x_{i j}^{R}\right) \leq 0, j=1, \ldots, n, j \neq k
\end{aligned}
$$

$v_{i}^{L}-v_{i}^{M} \leq 0,1 \leq i \leq m$

$v_{i}^{R}-v_{i}^{M} \leq 0,1 \leq i \leq m$

$v_{i}^{L}, v_{i}^{R}, v_{i}^{M} \geq 0,1 \leq i \leq m$

$u_{r}^{L}-u_{r}^{M} \leq 0,1 \leq r \leq s$

$u_{r}^{R}-u_{r}^{M} \leq 0,1 \leq r \leq s$

$u_{r}^{L}, u_{r}^{R}, u_{r}^{M} \geq 0,1 \leq r \leq s$

The dual of model (8) is

$0 \leq v_{i}^{L}, v_{i}^{R} \leq v_{i}^{M}, 1 \leq i \leq m$

$0 \leq u_{r}^{L}, u_{r}^{R} \leq u_{r}^{M}, 1 \leq r \leq s$ 
$\min \theta$

$$
\text { s.t. }
$$

$\theta x_{i k}^{L}-\sum_{j=1, j \neq k}^{n} \lambda_{j} x_{i j}^{L}+\dot{v}_{i} \geq 0, i=1, \ldots, m$

$\theta x_{i k}^{M}-\sum_{j=1, j \neq k}^{n} \lambda_{j} x_{i j}{ }^{M}-\dot{v}_{i}-\ddot{v}_{i} \geq 0, i=1, \ldots, m$

$\theta x_{i k}^{R}-\sum_{j=1, j \neq k}^{n} \lambda_{j} x_{i j}^{R}+\ddot{v}_{i} \geq 0, i=1, \ldots, m$

$\sum_{j=1, j \neq k}^{n} \lambda_{j} y_{r j}^{L}+\dot{u}_{r} \geq y_{r k}^{L}, r=1, \ldots, s$

$\sum_{j=1, j \neq k}^{n} \lambda_{j} y_{r j}^{M}-\dot{u}_{r}-\ddot{u}_{r} \geq y_{r k}^{M}, r=1, \ldots, s$

$\sum_{j=1, j \neq k}^{n} \lambda_{j} y_{r j}^{R}+\ddot{u}_{r} \geq y_{r k}^{R}, r=1, \ldots, s$

$\lambda_{j} \geq 0, j=1, \ldots, n, j \neq k$

$\dot{v}_{i} \geq 0, i=1, \ldots, m$

$\ddot{v}_{i} \geq 0, i=1, \ldots, m$

$\dot{u}_{r} \geq 0, r=1, \ldots, s$

$\ddot{u}_{r} \geq 0, r=1, \ldots, s$

Model (9) guarantees that the weight of the midpoint of a fuzzy number is not less than the weights of the right-end and left-end points of a fuzzy number. To ensure that the weight of the midpoint of a fuzzy number is greater than the weights of the right-end and left-end points of a fuzzy number, model (8) can be modified as follows:

$$
\begin{aligned}
& \max \sum_{r=1}^{s}\left(u_{r}^{L} y_{r k}^{L}+u_{r}^{M} y_{r k}^{M}+u_{r}^{R} y_{r k}^{R}\right) \\
& \text { s.t. } \\
& \sum_{i=1}^{m}\left(v_{i}^{L} x_{i k}^{L}+v_{i}^{M} x_{i k}^{M}+v_{i}^{R} x_{i k}^{R}\right)=1 \\
& \quad \sum_{r=1}^{s}\left(u_{r}^{L} y_{r j}^{L}+u_{r}^{M} y_{r j}^{M}+u_{r}^{R} y_{r j}^{R}\right) \\
& \quad-\sum_{i=1}^{m}\left(v_{i}^{L} x_{i j}^{L}+v_{i}^{M} x_{i j}^{M}+v_{i}^{R} x_{i j}^{R}\right) \leq 0, j=1, \ldots, n, j \neq k \\
& v_{i}^{L}-v_{i}^{M} \leq-\varepsilon, 1 \leq i \leq m \\
& v_{i}^{R}-v_{i}^{M} \leq-\varepsilon, 1 \leq i \leq m \\
& v_{i}^{L}, v_{i}^{R}, v_{i}^{M} \geq 0,1 \leq i \leq m \\
& u_{r}^{L}-u_{r}^{M} \leq-\varepsilon, 1 \leq r \leq s \\
& u_{r}^{R}-u_{r}^{M} \leq-\varepsilon, 1 \leq r \leq s \\
& u_{r}^{L}, u_{r}^{R}, u_{r}^{M} \geq 0,1 \leq r \leq s
\end{aligned}
$$

where $\varepsilon$ is a small positive number, which guarantees that the weight of the midpoint is larger than those of the end points.
Table 1. Linguistic variables and their corresponding normal triangular interval-valued fuzzy numbers.

\begin{tabular}{ll}
\hline Linguistic variables & Fuzzy numbers \\
\hline Very Poor & $(1,1,2)$ \\
Poor & $(1,2,3)$ \\
Medium Poor & $(2,3,4)$ \\
Fair & $(3,4,5)$ \\
Medium Good & $(4,5,6)$ \\
Good & $(5,6,7)$ \\
Very Good & $(6,7,7)$ \\
\hline
\end{tabular}

\section{Numerical examples}

To verify the validity of the new proposed model, we used the dataset of Lee et al., [18]. Our example derives from actual production operations in Taiwan's textile industry. In this example, assume that there are three suppliers being evaluated under the following evaluation criteria: price (C1), quality (C2), delivery (C3), flexibility (C4), and service (C5). Among these criteria, $\mathrm{C} 1$ is the cost criterion, whereas C2, C3, C4, and C5 are the benefit criteria. The linguistic variables and their corresponding normal triangular interval-valued fuzzy numbers are summarized in Table 1 (corresponding schematic diagram see Fig. 1).

The operating performance matrix of the selected suppliers is summarized in Table 2. Under the model (6), this study computes the efficiency for each evaluated supplier, and the comparison of summary evaluation results is shown in Table 3. Note that the value of $\epsilon$ of model (6) is 0.001 .

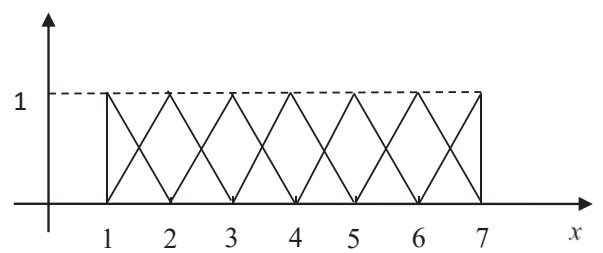

Fig. 1. A normal triangular interval-valued fuzzy number.

Table 2. Performance matrix of the suppliers and evaluation results.

\begin{tabular}{llllll}
\hline Supplier & C1 & C2 & C3 & C4 & C5 \\
\hline Supplier 1 & High & Good & Very Good & Good & Fair \\
Supplier 2 & Low & Very Good & Good & Good & Good \\
Supplier 3 & Fair & Medium Good & Good & Fair & Good \\
\hline
\end{tabular}

Table 3. The comparison of summary evaluation results.

\begin{tabular}{llllll}
\hline Supplier & Lee et al., [18] & & & Model (10) & \\
\cline { 2 - 3 } \cline { 5 - 6 } \cline { 5 - 6 } & Composite index & Rank & & Efficiency & Rank \\
\hline Supplier 1 & 0.27704 & 3 & & 0.45455 & 3 \\
Supplier 2 & 0.95638 & 1 & & 3.66667 & 1 \\
Supplier 3 & 0.29619 & 2 & & 0.55556 & 2 \\
\hline
\end{tabular}


Table 4. Numerical case with 9 suppliers.

\begin{tabular}{|c|c|c|c|c|c|c|c|}
\hline \multirow[t]{2}{*}{ Supplier } & \multirow{2}{*}{$\frac{\text { Cost Criterion }}{\mathrm{C} 1}$} & \multicolumn{4}{|l|}{ Benefit Criteria } & \multicolumn{2}{|l|}{ Results } \\
\hline & & $\mathrm{C} 2$ & $\mathrm{C} 3$ & $\mathrm{C} 4$ & $\mathrm{C} 5$ & Efficiency & Rank \\
\hline Supplier 1 & High & Medium Poor & Very Good & Fair & Medium Good & 0.435833 & 7 \\
\hline Supplier 2 & High & Medium Good & Poor & Good & Fair & 0.383308 & 8 \\
\hline Supplier 3 & High & Poor & Medium Good & Poor & Poor & 0.315397 & 9 \\
\hline Supplier 4 & Low & Very Poor & Very Good & Fair & Medium Good & 1.581926 & 2 \\
\hline Supplier 5 & Low & Medium Good & Medium Good & Very Good & Fair & 2.178400 & 1 \\
\hline Supplier 6 & Low & Fair & Medium Good & Very Poor & Very Good & 1.523733 & 3 \\
\hline Supplier 7 & Fair & Poor & Medium Good & Fair & Medium Good & 0.541530 & 5 \\
\hline Supplier 8 & Fair & Medium Good & Poor & Good & Fair & 0.554333 & 4 \\
\hline Supplier 9 & Fair & Medium Poor & Medium Good & Poor & Medium Good & 0.506924 & 6 \\
\hline
\end{tabular}

The comparison clearly revealed the consistency between the evaluation ranking under our fuzzy MCDM model and the results of Lee et al., [18]; which they obtained using their novel intervalvalued fuzzy number approach. In general, supplier 2 was clearly the best performing because it had extremely satisfactory performance (for the benefit criteria) at the lowest cost.

Furthermore, to evaluate the discriminative ability of the new proposed fuzzy MCDM model, a numerical example with nine suppliers was analyzed (Table 4). The two rightmost columns in Table 4 present the evaluation results and ranking.

Table 4 details the basic characteristics of each supplier, and the evaluation results are presented in the seventh and eighth columns from the left. Note that similar to model (6), in model (10), $\varepsilon=0.001$. In Table 4, our fuzzy MCDM model provided a clear ranking for all evaluated suppliers. According to the features of the super-efficiency DEA model, suppliers 4, 5, and 6 were all relatively efficient (all efficiency scores $>1$ ). If the traditional fuzzy DEA model is used, the evaluation results of the three suppliers are equal to 1 . In other words, our fuzzy MCDM model discriminates better than its traditional counterpart, allowing the most suitable supplier to be selected more efficiently. Furthermore, our model also discriminated between inefficient suppliers significantly better than its traditional counterpart does. For example, our model could differentiate between the operating performances of suppliers 7 and 9, which had similar criteria ratings. In particular, both suppliers were rated "Fair" under the cost criterion, "Medium Good" under two benefit criteria, and "Poor" under one benefit criteria; the suppliers differed with respect to only one benefit criterion, where suppliers 7 and 9 obtained the ratings of "Fair" and "Medium Poor," respectively.

\section{Conclusion}

Supplier selection is crucial to sustainable development and thus has been essential in supply chain management. A well-managed supply chain is crucial to ensuring the success of a company, particularly in gaining customer satisfaction. In modern supply chain management, enterprises have the central task of responding to the myriad of customer demands while reducing overall cost. To do so, the most suitable supplier must be selected. Having such a supplier can not only make an enterprise's logistics and transportation more efficient (e.g., in reducing the cost of inventory) but also guarantee the enterprise's sustainable growth and ability to gain customer trust and satisfaction. Therefore, managers must be equipped with the appropriate evaluation methods and standards when selecting the best supplier from a set of candidates.

Thus, to solve the supplier selection problem, this study proposes a new fuzzy MCDM model based on the fuzzy DEA model. Our model was demonstrated to have greater discrimination ability relative to its traditional counterpart for both efficient and inefficient DMUs. We hope that our model can provide scholars and managers with new insights into solving the MCDM problem in supplier selection.

\section{Acknowledgements}

This research is partially supported by the natural science research project of university in Jiangsu (19KJB120009), and National Natural Science Foundation of China (No.71801133). The authors are also grateful to the comments and suggestions provided by anonymous reviewers.

\section{References}

[1] Ho W, Ma X. The state-of-the-art integrations and applications of the analytic hierarchy process. Eur J Oper Res 2018; 267(2):399-414.

[2] Wang B, Xie HL, Ren HY, Li X, Chen L, Wu BC. Application of AHP, TOPSIS, and TFNs to plant selection for phytoremediation of petroleum-contaminated soils in shale gas and oil fields. J Clean Prod 2019;233:13-22. 
[3] Javid N, Khalili-Damghani K, Makui A, Abdi F. Multiobjective flexibility-complexity trade-off problem in batch production systems using fuzzy goal programming. Expert Syst Appl 2020;148:113266.

[4] Jiang H, Kwong CK, Chan CY, Yung KL. A multi-objective evolutionary approach for fuzzy regression analysis. Expert Syst Appl 2019;130:225-35.

[5] Yu H, Solvang WD. A fuzzy-stochastic multi-objective model for sustainable planning of a closed-loop supply chain considering mixed uncertainty and network flexibility. J Clean Prod 2020;266. Article 121702.

[6] Luo S, Zhang L, Fan Y. Energy-efficient scheduling for multiobjective flexible job shops with variable processing speeds by grey wolf optimization. J Clean Prod 2019;234:1365-84.

[7] Yang Y, Yang B, Wang S, Jin T, Li S. An enhanced multiobjective grey wolf optimizer for service composition in cloud manufacturing. Appl Soft Comput 2020;87. Article 106003.

[8] Chen P. Effects of normalization on the entropy-based TOPSIS method. Expert Syst Appl 2019;136:33-41.

[9] Lupo T, Bellomo E. DINESERV along with fuzzy hierarchical TOPSIS to support the best practices observation and service quality improvement in the restaurant context. Comput Ind Eng 2019;137. Article 106046.

[10] Boyd GA, Lee JM. Measuring plant level energy efficiency and technical change in the US metal-based durable manufacturing sector using stochastic frontier analysis. Energy Econ 2019;81:159-74.

[11] Stead AD, Wheat P. The case for the use of multiple imputation missing data methods in stochastic frontier analysis with illustration using English local highway data. Eur J Oper Res 2020;280(1):59-77.

[12] Tavakoli IM, Mostafaee A. Free disposal hull efficiency scores of units with network structures. Eur J Oper Res 2019; 277(3):1027-36.

[13] Mostafaee A, Soleimani-damaneh M. Global sub-increasing and global sub-decreasing returns to scale in free disposal hull technologies: Definition, characterization and calculation. Eur J Oper Res 2020;280(1):230-41.

[14] Fukuyama H, Matousek R, Tzeremes NG. A Nerlovian cost inefficiency two-stage DEA model for modelling banks' production process: evidence from the Turkish banking system. Omega; 2020. Article 102198.

[15] Gan GY, Lee HS. An alternative MILP-DEA model to choose efficient unit without explicit inputs. Ann Oper Res 2019; 278(1-2):379-91.

[16] Lee HS. Efficiency decomposition of the network DEA in variable returns to scale: An additive dissection in losses. Omega; 2020. Article 102212.

[17] Lim S, Zhu J. Primal-dual correspondence and frontier projections in two-stage network DEA models. Omega 2019; 83:236-48.

[18] Lee CS, Chung CC, Lee HS, Gan GY, Chou MT. An intervalvalued fuzzy number approach for supplier selection. J Mar Sci Technol 2016;24(3):384-9.
[19] Fu YK. An integrated approach to catering supplier selection using AHP-ARAS-MCGP methodology. J Air Transport Manag 2019;75:164-9.

[20] Haeri SAS, Rezaei J. A grey-based green supplier selection model for uncertain environments. J Clean Prod 2019;221: 768-84.

[21] Kilic HS, Yalcin AS. Modified two-phase fuzzy goal programming integrated with IF-TOPSIS for green supplier selection. Appl Soft Comp 2020;93:106371.

[22] Dobos I, Vörösmarty G. Green supplier selection and evaluation using DEA-type composite indicators. Int J Prod Econ 2014;157:273-8.

[23] Jauhar SK, Pant M. Integrating DEA with DE and MODE for sustainable supplier selection. J Math Comput Sci 2017;21: 299-306.

[24] Dobos I, Vörösmarty G. Inventory-related costs in green supplier selection problems with Data Envelopment Analysis (DEA). Int J Prod Econ 2019;209:374-80.

[25] Davoudabadi R, Mousavi SM, Sharifi E. An integrated weighting and ranking model based on entropy, DEA and PCA considering two aggregation approaches for resilient supplier selection problem. J Math Comput Sci 2020;40: 101074.

[26] Karsak EE, Dursun M. An integrated supplier selection methodology incorporating QFD and DEA with imprecise data. Expert Syst Appl 2014;41(16):6995-7004.

[27] Azadi M, Jafarian M, Farzipoor Saen R, Mirhedayatian SM. A new fuzzy DEA model for evaluation of efficiency and effectiveness of suppliers in sustainable supply chain management context. Comput Oper Res 2015;54:274-85.

[28] Cheng Y, Peng J, Zhou Z, Gu X, Liu W. A hybrid DEAadaboost model in supplier selection for fuzzy variable and multiple objectives. IFAC-PapersOnLine 2017;50(1): 12255-60.

[29] Rashidi K, Cullinane K. A comparison of fuzzy DEA and fuzzy TOPSIS in sustainable supplier selection: Implications for sourcing strategy. Expert Syst Appl 2019;121:266-81.

[30] Banker RD, Charnes A, Cooper WW. Some models for estimating technical and scale inefficiencies in data envelopment analysis. Manag Sci 1984;30:1078-92.

[31] Charnes A, Cooper WW, Rhodes E. Measuring the efficiency of decision making units. Eur J Oper Res 1978;2:429-44.

[32] Barak S, Dahooei JH. A novel hybrid fuzzy DEA-Fuzzy MADM method for airlines safety evaluation. J Air Transport Manag 2018;73:134-49.

[33] Chen L, Wang YM. Data envelopment analysis cross-efficiency model in fuzzy environments. J Intell Fuzzy Syst 2016; 30(5):2601-9.

[34] Hatami-Marbini A, Emrouznejad A, Tavana M. A taxonomy and review of the fuzzy data envelopment analysis literature: Two decades in the making. Eur J Oper Res 2011;214:457-72.

[35] Kao C, Liu ST. Fuzzy efficiency measures in data envelopment analysis. Fuzzy Sets and Systems 2000;113:427-37.

[36] Liu ST. A fuzzy DEA/AR approach to the selection of flexible manufacturing systems. Comput Ind Eng 2008;54:66-76. 